A fibre-optic temperature sensor based on the deposition of a thermochromic material on an adiabatic taper

This content has been downloaded from IOPscience. Please scroll down to see the full text. 2004 Meas. Sci. Technol. 15353

(http://iopscience.iop.org/0957-0233/15/2/006)

View the table of contents for this issue, or go to the journal homepage for more

Download details:

IP Address: 147.96.14.15

This content was downloaded on 03/12/2013 at 17:08

Please note that terms and conditions apply. 


\title{
A fibre-optic temperature sensor based on the deposition of a thermochromic material on an adiabatic taper
}

\author{
N Díaz-Herrera ${ }^{1}$, M C Navarrete $^{1}$, O Esteban $^{2}$ and \\ A González-Cano ${ }^{3,4}$ \\ ${ }^{1}$ Departamento de Óptica, Facultad de Ciencias Físicas, Universidad Complutense de \\ Madrid, Ciudad Universitaria, s/n 28040, Madrid, Spain \\ ${ }^{2}$ Departamento de Electrónica, Universidad de Alcalá de Henares, Alcalá de Henares, \\ Madrid, Spain \\ ${ }^{3}$ Departamento de Óptica, Escuela Universitaria de Óptica, Universidad Complutense de \\ Madrid, Arcos de Jalón, s/n 28037, Madrid, Spain \\ E-mail: agus@ @fis.ucm.es
}

Received 2 July 2003, in final form and accepted for publication

7 November 2003

Published 11 December 2003

Online at stacks.iop.org/MST/15/353 (DOI: 10.1088/0957-0233/15/2/006)

\begin{abstract}
A fibre-optic sensor has been developed for the measurement of temperature, especially of liquids. The device is conceived as part of an all-optical CTD probe for the control of the physical parameters of a marine medium. The dependence on temperature of the optical properties (specifically, absorbance) of a thermochromic material, namely lophine (2,4,5-triphenylimidazole), is the basis of the sensor. The sensor presents some significant differences with respect to other similar sensors proposed in the literature: the use of adiabatic, long, tapered optical fibres with adjustable geometric parameters; the use of LED illumination in the $800 \mathrm{~nm}$ range; improvements in the deposition technique, etc. The sensors show a linear behaviour over the desired temperature range, and their sensitivity is high. Also, the dependence of the response of the sensor with variations of the geometry of the tapers is discussed. Specifically, we have performed measurements with different diameters of the taper waist, and we show the dependence of the slope of the response curve with that parameter.
\end{abstract}

Keywords: fibre-optic sensors, temperature measurement, tapered optical fibres, lophine

(Some figures in this article are in colour only in the electronic version)

\section{Introduction}

Temperature is, obviously, a very relevant physical parameter, whose accurate determination is important in many contexts. Specifically, in environmental measurements, like those involved in the control of the physical parameters of a marine medium, the temperature is a property routinely measured by different techniques, some of them optical. In the framework

4 Author to whom any correspondence should be addressed. of a European Research Project (MISPEC, Multi-parametric in situ spectroscopic system for coastal monitoring, EVK3CT2000-00519), our team has been developing the new concept of a CTD probe (here CTD stands for conductivitytemperature-depth) composed only of fibre-optic sensors, one of which (the salinity one) has been successfully demonstrated some years ago [1]. In principle, the use of optical techniques, in general, presents some advantages with respect to electrical measurements, since these are always potentially subject to electrical interferences, which are possible when the 
sensor is working together with other electrical measuring devices.

Different optical techniques may be used to measure the temperature in the context we have defined (namely, real measuring conditions in a marine medium). Among them, we are interested specifically in the use of fibre-optic sensors. There exist in the literature numerous optical fibre sensors for temperature measurements, based on different principles [2-17]. The use of optical fibre sensors presents some specific advantages, namely, the accessibility to difficult places, the potential simplicity of the design (especially when the same fibre is used both as sensing element and information transmission channel), the wide range of temperatures that can be covered by choosing the appropriate parameters in the design, etc. It must be taken into account that the sea is a hostile medium, and that some laboratory techniques are not too well adapted to real measurement conditions. For instance, the feasibility of a robust arrangement of the device is a key feature, and the simplicity of the concept is a good way to provide that robustness. Using non-sophisticated devices also reduces costs and fragility. The sensor depicted in this paper presents some of these advantages, being simple, compact, easy to produce, and with an accuracy and a dynamical range enough for the above defined objective.

The device is based on the deposition of a thermochromic material, lophine (2,4,5-triphenylimidazole), over a tapered optical fibre. This material is hydrophobic, which makes it an interesting candidate for any temperature measurement in liquids. The scheme we present has been successfully proposed in a somewhat different form by FernándezValdivielso et al, from the Universidad Pública de Navarra (Spain) [18]. We have introduced significant modifications in the technique. The most important one is the possibility of controlling the geometric parameters of the tapers, which are adiabatic, long ones. We have also obtained higher sensitivities and a linear response over the desired range, which is defined by the requirements of the sensor. In this way, we have obtained an operative sensor which can be used as the temperature component of an all-optical CTD probe.

\section{The sensor concept}

A thermochromic material changes its optical properties (specifically, spectral absorbance) as a response to temperature variations. In the case of lophine, its particular chemical configuration is affected by temperature. The result is an increase in the absorption of light in a wide spectral range. This effect has been used as the physical principle of different fibre-optic sensors [10, 16, 18].

In general, we should provide some form of coupling between a guided field and the thermochromic material. Both reflection and transmission schemes are possible. We can dip the end of an optical fibre in lophine and study the variations of the reflectance of the device. Sensors of this type have been successfully demonstrated $[16,18]$. When using reflectance as the measured parameter, only one fibre is used for the input and output light. However, one always needs to use couplers or optical circulators to perform the measurements, thus somehow complicating the set-up. The deposition of the lophine by dipping the end of the fibre

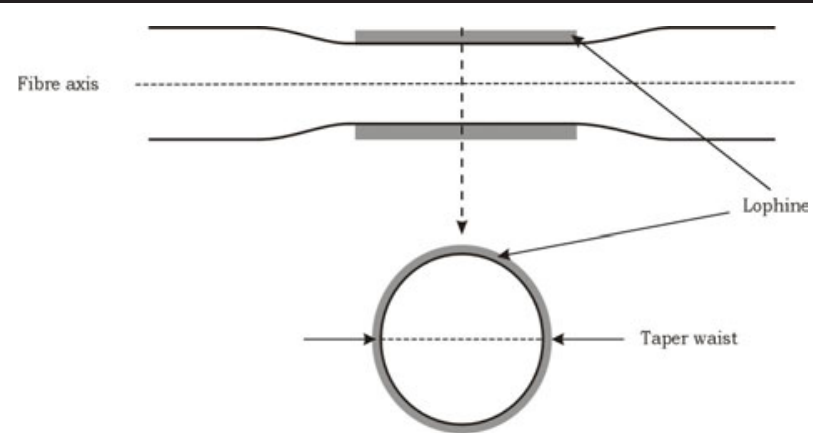

Figure 1. The scheme of the sensor concept. The material (lophine) is deposited on the tapered region of the fibre. A longitudinal profile (top) and cross section (bottom) are shown.

to produce reflective sensors is also itself a problem. We discuss the importance of the deposition technique in the next paragraph: the repeatability of the measurements is strongly influenced by the deposition. Controlling the thickness of the deposited layer is difficult in the production of the reflective sensors and some effects similar to 'beats' have been observed in their response curve.

We can also use a transmissive scheme, arranging the system to permit the evanescent field of the modes guided by the fibre to interact with the thermochromic material, and studying then the variation of the power transmitted by the fibre. Among the different mechanisms that permit such an evanescent coupling, we have chosen to use tapered optical fibres.

In these devices the field guided by the fibre is coupled into the taper, which is a narrowing produced in the fibre by heating and stretching it. The fabrication of tapers requires a specific technique, which is depicted in the next paragraph. In the tapered region the field is guided by the cladding, since the core has almost collapsed in the narrowing process, and therefore it is in principle accessible. If we deposit on the taper a material, or, most generally, if we have an outer medium in contact with the cladding that shows some variation of its optical parameters, this variation will affect the transmission of the field by the tapered fibre. In our case, this variation is a change in the absorption of light by lophine, which is dependent on temperature. Therefore, the optical power transmitted by the tapered fibre will be a function of temperature.

Transmission sensors based on short tapers have also been depicted in the literature. We propose, as we have said before, to use adiabatic tapers instead of short ones, this being one of the main features of our device. We use only one fibre that goes from the source to the detector, as is shown in figure 1 . The fibre is used both as a transmission channel and as a sensor. No couplers or any other elements are used, simply a source, the fibre with the material deposited on the taper, and the detector. This simplicity of concept is, in our view, a key feature of the proposed device.

We use an LED as the illuminating source, which is very convenient from the practical point of view, because of its compactness and easy operation. We have also used a singlemode optical fibre (commercial 3M-FSSN 4224 fibre, core diameter $5 \mu \mathrm{m}$, cladding diameter $125 \mu \mathrm{m}$ ), for transmission in the $820-850 \mathrm{~nm}$ range, NIR region. 


\section{The production of tapered optical fibres}

Some years have passed since the introduction of the taper concept, and devices based on tapered optical fibres have been used for very different purposes [19-21]. Also, the behaviour of light in these structures has been theoretically studied and conveniently modelled $[22,23]$. Since the very beginning, a major distinction has been established between adiabatic and non-adiabatic tapers. The adiabaticity of the tapers depends mainly on the fabrication process. If the taper is long enough, and the transition between the unaltered fibre and the tapered region is smooth enough, the loss of optical power in this coupling can be very low, and then we speak of adiabatic tapers. If these conditions are not fulfilled, the coupling is less effective, as it is the case with short, abrupt tapers, normally produced with a splicer, where the coupling between the unaltered region and the taper is not energetically effective, and the transmission losses are high. On the other hand, techniques like the so-called travelling burner one, proposed by Kenny et al [24], permit us to smoothly stretch the fibre while heating it, and, in principle, can provide almost perfect coupling between the different regions of the device. These adiabatic tapers are long, and their geometrical parameters can be adjusted by modifying the conditions of the heating-andstretching process. The most important parameters are taper length and taper waist diameter. The profile of the transition region between the unaltered region and the taper waist can also be defined.

These degrees of freedom in the design are very important while developing sensors for specific requirements. In our case, we have used the set-up shown in figure 2, and have obtained long, adiabatic tapers (lengths typically of 5-10 mm, losses as low as $0.1 \mathrm{~dB})$, with varying waist diameters (35$60 \mu \mathrm{m})$. These parameters can be adjusted in our case by controlling the movement of the heater, both in range and speed. The set-up is automatically operated, so the movement of the heater and of the drivers that stretch the fibres is conveniently synchronized. The operation is based on theoretical models which predict the geometrical parameters of the tapers from these controllable magnitudes, and the agreement between the predictions and the results are very good, as shown in figure 3 , where a real taper is compared to the predicted profile.

The possibility of using adiabatic tapers is important in our case for two main reasons. First, we have lower initial losses than in other techniques, so the losses in our device depend on the variation of temperature, once the lophine is deposited, and the output signal is correspondingly higher. Second, and most important, the increase of the interaction region, even when working with very narrow waists, enhances the response of the sensor, increasing its sensitivity, as will be shown when we present the results.

\section{The deposition technique}

Working with lophine is not an easy task. Simply dipping the tapers in lophine is not a real option, especially when we are speaking of long tapers. At environmental temperatures, lophine is a solid substance, so we must fuse it first to deposit it on the taper. The fusion temperature of lophine is high, so,

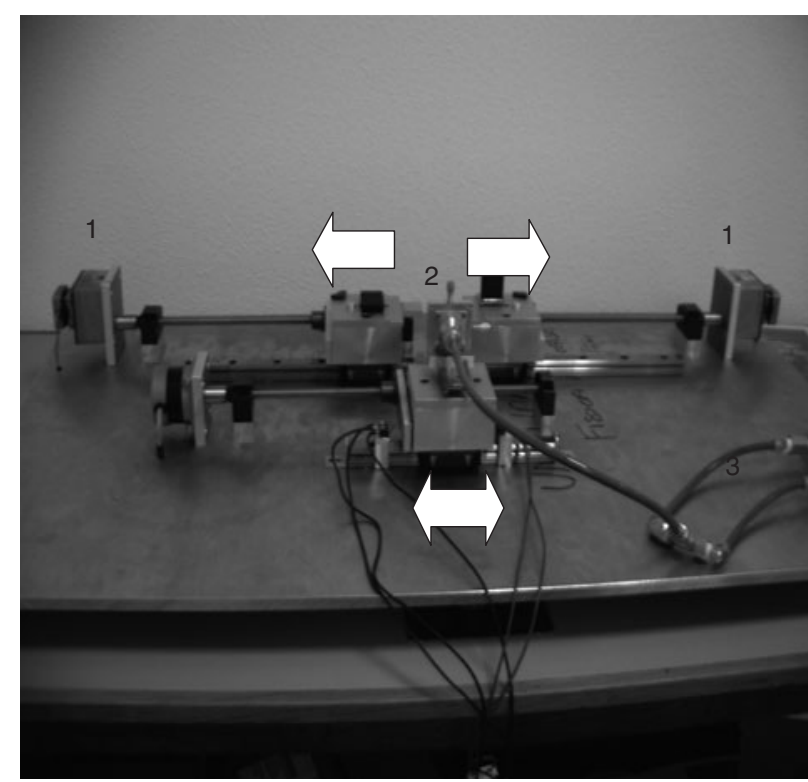

Figure 2. A view of the set-up for production of long tapers (travelling-burner technique). Two motors (1) stretch the fibre while it is heated by a specially designed heater (2). The gas inlet appears on the right (3).

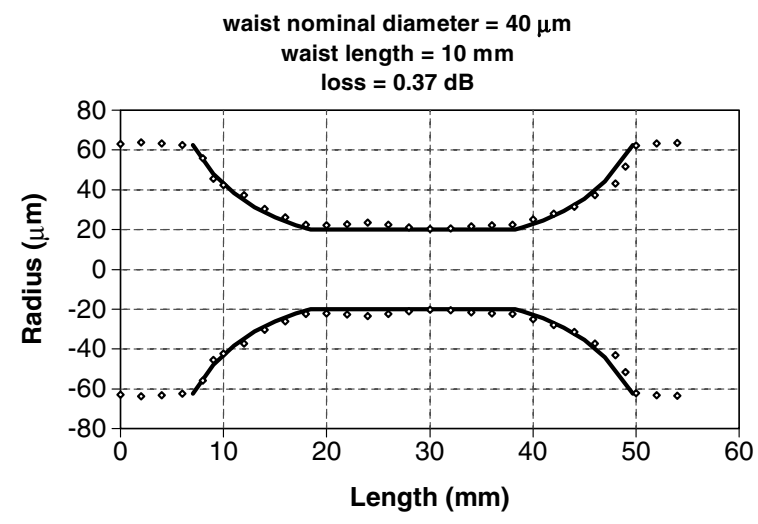

Figure 3. The profile of a produced taper. Dots correspond to experimental measurements with an optical microscope. The continuous line corresponds to the profile predicted by a theoretical model. The parameters of the taper are also shown in the figure.

as the taper is fragile due to its very small diameter, it can be broken if hot lophine is poured upon it. But lophine is also quickly solidified, so there is not time to pour it conveniently. The process is very critical, since the performance of the sensors strongly depends on a good deposition, which should assure a real coupling between the fibre and the absorptive region. The deposited thickness is, in any case, big (of the order of $1 \mathrm{~mm}$ ), so we are working with a bulk material. The most important issue is the repeatability of the technique. To cope with these problems, we assayed different configurations for the deposition. For instance, we poured lophine on a taper supported by a glass plate or we created a lophine cylinder by not using any support. Finally we developed the deposition technique which is shown in figure 4. As can be seen, the taper is placed on a groove on a metal plate, and lophine is poured onto it by using a canal. In this way we optimize the time of deposition, keeping then the lophine in its liquid state, 


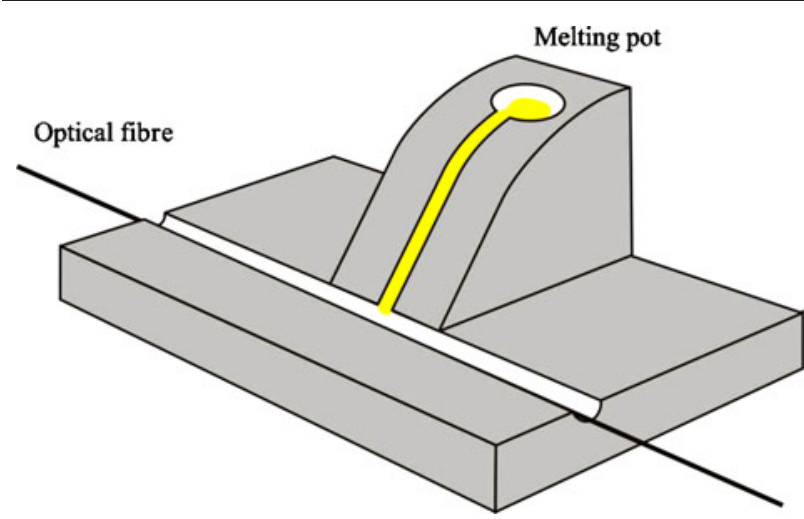

Figure 4. The technique for the deposition of lophine. The taper is placed on a plate with a groove. Melted lophine is poured on it.

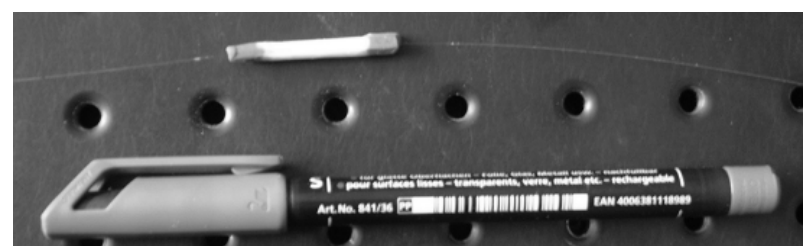

Figure 5. Lophine deposited on a taper. This is one of several configurations we used for the sensor. A pen is included in the picture for comparison of sizes (the size of the sensor is about $2 \mathrm{~cm}$ )

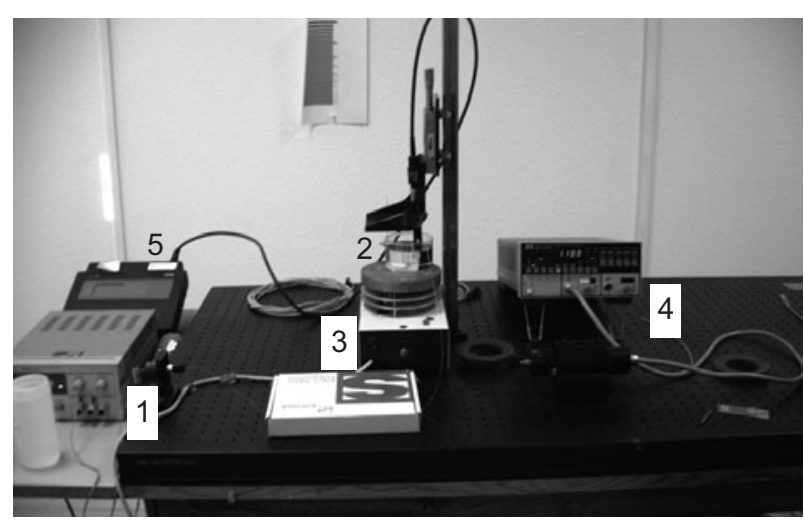

Figure 6. The experimental set-up for the characterization of the sensors. Light coming out of the LED (1) is guided to the sensor (2), which is submerged in a recipient with water, placed on a heater (3). The output signal is measured by an optical power meter (4). The temperature and the salinity of the water are controlled during the process (5)

and we also improve the homogeneity of the deposited layer. With this technique we obtain the best repeatability, and the devices produced with it show the best behaviour. A picture of a sensor is shown in figure 5 .

\section{Experimental results}

The experimental set-up is very simple and is shown in figure 6. Light coming from the LED (peak wavelength $820 \mathrm{~nm}$ ) is coupled into the single-mode fibre, and arrives at the tapered region, where it interacts with the thermochromic material. The output power transmitted by the fibre after this interaction is measured with a commercial HP power meter.

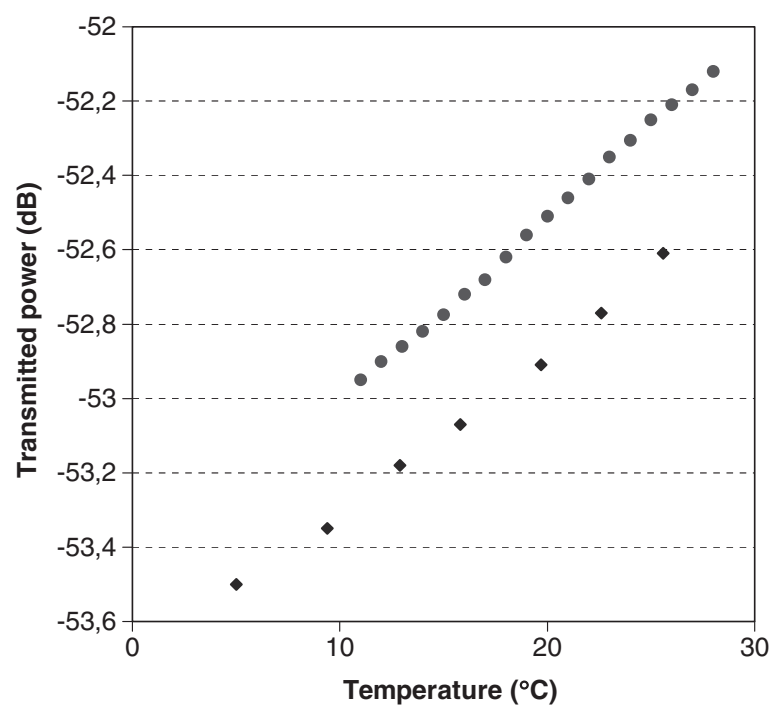

Figure 7. The response of the sensor to temperature variations. Circles correspond to measurements with our laboratory set-up (sensor in water) and diamonds to measurements performed in a climatic chamber (sensor in air)

The sensor is submerged in water, which is heated by a hot plate in a controlled way from 10 to $40^{\circ} \mathrm{C}$. We have performed both ascendant and descendant measurement series. The readings of the sensor response are made in a continuous way, since the heating of the system is progressive. But we have also used a climatic chamber to check the performance of the sensor in a dry environment and with controlled, stable temperatures.

In figure 7 we show a typical behaviour of the sensors, comparing the measurements in water (circles) and in the climatic chamber (diamonds). Although we obtain a different signal level, the slope is the same. We can see that the responses are linear, and that this linearity covers the region of interest for the requirements of the sensor. The dynamic range is also good enough. We have obtained this kind of result by varying the taper parameters and the technique of deposition, and consider that the suitability of the proposed approach for temperature sensing is proved. One important point is the sensitivity of the measurements, which is quite high compared to other sensors of this same type in the literature (about $0.05 \mathrm{~dB}$ per degree). This confirms the importance of using adiabatic tapers.

In figures 8 and 9 we study another interesting question: the dependence of the behaviour of the sensors on the waist diameter. As we said before, one very convenient feature of our proposal is the possibility of varying the parameters of the sensor. In figure 8 the lower curve (triangles correspond to experimental points) shows the response of a $60 \mu \mathrm{m}$-waist taper, and the upper curve (diamonds) that of a $45 \mu \mathrm{m}$-waist taper. As can be seen, the linearity is good in both cases, and the response of the sensors (the slope of the curves) is clearly dependent on the waist diameter of the tapers. In figure 9 the waists are $40 \mu \mathrm{m}$ (lower curve, squares) and $35 \mu \mathrm{m}$ (upper curve, circles). The tendency towards a steeper slope (and a higher sensitivity) as a function of waist diameter is confirmed. We can expect such a behaviour if we take into account that a smaller waist favours the interaction of the guided field with the deposited material. 
A fibre-optic temperature sensor based on the deposition of a thermochromic material

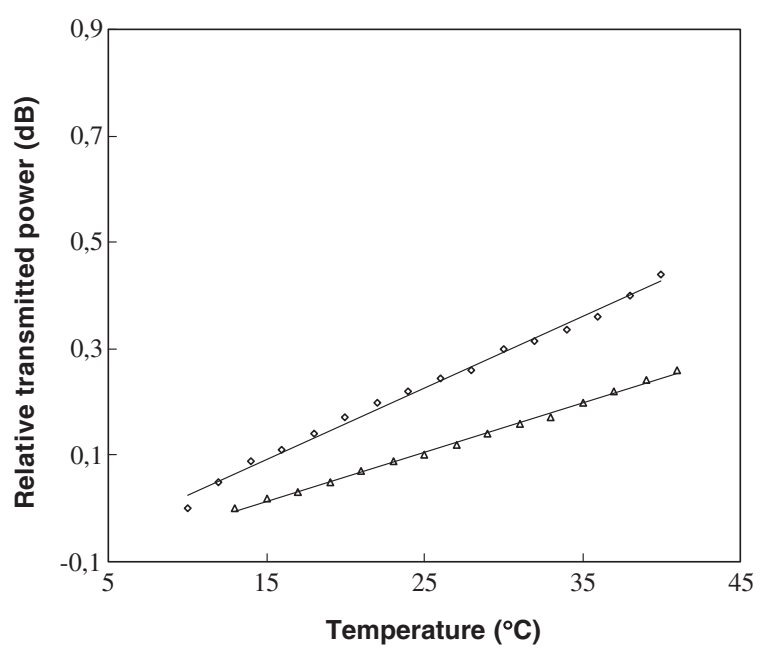

Figure 8. The dependence of the sensor response on the diameter of the taper waist. The lower curve (diamonds) corresponds to a waist of $60 \mu \mathrm{m}$; the upper curve (triangles) corresponds to a $45 \mu \mathrm{m}$ waist.

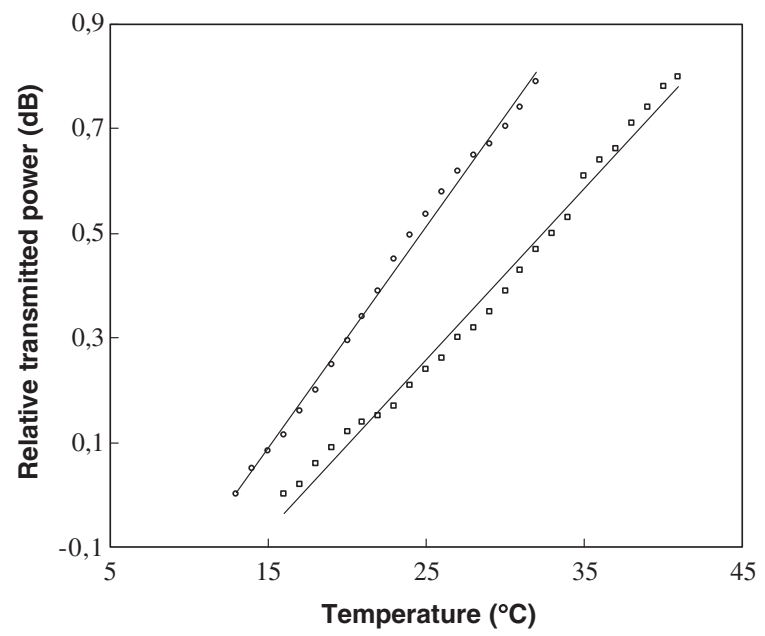

Figure 9. The dependence of the sensor response on the diameter of the taper waist for thin waists. The lower curve (squares) corresponds to a waist of $40 \mu \mathrm{m}$; the upper curve (circles) corresponds to a waist of $35 \mu \mathrm{m}$. An increase of the slope is clearly shown.

As a simple way to easily visualize the dependence of the slope with waist diameter, we show in figure 10 the values of the slope versus the diameters. The increase in the slope, and consequently of the sensitivity of the sensor, is remarkable, so we can adjust the slope to the desired value by simply varying the taper waist, or we can favour the design of sensors with waists of about $35 \mu \mathrm{m}$ if sensitivity is a major requirement. Interestingly, this is more or less the thickness that one can obtain for short, non-adiabatic tapers if one uses a splicer to produce the tapers, but our tapers are much longer, so the sensitivity is significantly increased, as compared to other sensors based on short tapers proposed in the literature [16, 18].

\section{Conclusions}

We have shown the feasibility of a temperature sensor based on the deposition of a thermochromic material on an adiabatic tapered optical fibre. This device can be used as part of an

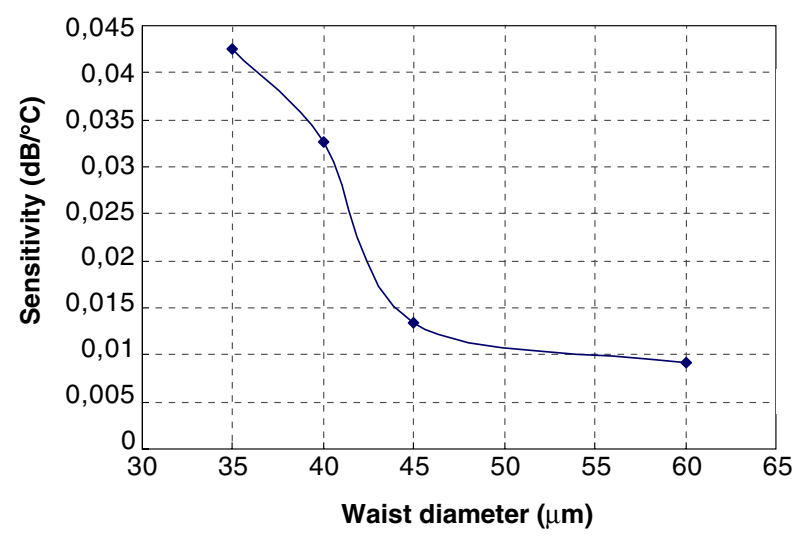

Figure 10. The dependence of the sensitivity of the sensor (slope of the response curves) on the waist diameter.

all-optical CTD probe for marine measurements, an important device from the environmental point of view. We have used a flexible production set-up to automatically vary the geometrical parameters of the tapers, while keeping their adiabaticity, and we have improved the deposition technique of the material, obtaining higher repeatability. The results obtained are good in terms of linearity, dynamic range and sensitivity. The dependence of these results on the parameters of the tapers has been studied, and it has been shown that a narrower taper waist increases significantly the slope of the response curve, and, consequently, the sensitivity of the system. We can obtain a sensitivity of about $0.05 \mathrm{~dB}^{\circ} \mathrm{C}^{-1}$ for narrow tapers, so, even with a quite simple detection scheme, a resolution of the order of $0.2^{\circ} \mathrm{C}$ is possible.

The characteristics of the sensor suggest to us that in principle a very easy design for a marine device can be possible. The exposition of the sensitive region to water is not a problem, since lophine is hydrophobic, and the fragile taper is protected with this same lophine. The dimensions of the sensor are very small, so we can produce a very compact measuring device, compatible with the requirements of the optical CTD probe (ruggedness; possibility of accessing to difficult places; real-time, in situ measurements; immunity to electrical interferences, etc). The use of a very common illumination source and detector, and the independence of light polarization are also very important features. For all these reasons, we can conclude that the proposed sensor can be used as a component of a real, operative optical CTD probe.

\section{Acknowledgments}

This work has been partially supported by European Project MISPEC, contract number EVK3-CT2000-00519 and Spanish Project OPTIMA, Programa Nacional de Recursos Naturales, MCYT, ref. REN 2001-1495. We are indebted to C FernándezValdivielso, F J Arregui and I Matías of the Universidad Pública de Navarra (Spain) for their help and support.

\section{References}

[1] Esteban Ó, Navarrete M C, González-Cano A and Bernabeu E 1999 Measurement of the degree of salinity of water with a fiber-optic sensor Appl. Opt. 38 5267-71

[2] Dakin J and Culshaw B 1989 Systems and applications Optical Fiber Sensors vol 2 (Boston, MA: Artech House) 
[3] Varney M S (ed) 2000 Chemical Sensors in Oceanography (London: Taylor and Francis)

[4] Grattan K T V and Zhang Z Y 1995 Fiber Optic Fluorescence Thermometry (London: Chapman and Hall)

[5] Datta P, Matías I, Aramburu C, Bakas A, López-Amo M and Otón J M 1996 Tapered optical-fiber temperature sensor Microw. Opt. Technol. Lett. 11 93-5

[6] Yan J and Pang S 1997 Temperature based on etched optical fiber with a metal coating Int. J. Infrared Millim. Waves 18 1423-30

[7] Cortés R, Khomenko A V, Starodumov A N, Arzate N and Zenteno L A 1998 Interferometric fiber-optic temperature sensor with spiral polarization couplers Opt. Commun. 154 268-72

[8] Yuan L-B, Zhou L-M and Wu Jing-seng 2000 Fiber optic temperature sensor with duplex Michelson interferometric technique Sensors Actuators A 86 2-7

[9] Zhao W, Claus R O, Cooper K, Liu Y, Arregui F J and Matias I R 2000 Self-assembled nanostructured optical fiber temperature sensors 14th Int. Conf. on Optical Fiber Sensors ed A G Mignani and H C Lefèvre Proc. SPIE 4815 21-4

[10] Fernández-Valdivielso C, Matías I R, Madrid A and Arregui F J 2000 An optical fiber temperature sensor based on thermochromic effect for under-water applications Proc. SPIE 4185 146-9

[11] Khomenko A V, García-Weidner A, Tapia-Mercado J and García-Zárate M A 2001 High-resolution fast response using a twin interferometric temperature sensor $O p t$. Commun. 198 29-35

[12] Canning J, Sommer K and Englund M 2001 Fibre gratings for high temperature sensor applications Meas. Sci. Technol. 12 824-8
[13] Zhao Y and Liao Y 2002 Novel optical fiber sensor for simultaneous measurement of temperature and salinity Sensors Actuators B 86 63-7

[14] Khaliq S, James S W and Tatam R P 2002 Enhanced sensitivity fibre optic long period grating temperature sensor Meas. Sci. Technol. $13792-5$

[15] Seat H C and Sharp J H $2003 \mathrm{Er}^{3+}+\mathrm{Yb}^{3+}$-codoped $\mathrm{Al}_{2} \mathrm{O}_{3}$ crystal fibres for high-temperature sensing Meas. Sci. Technol. 14 279-85

[16] Fernández-Valdivielso C, Matías I R and Arregui F J 2002 Simultaneous measurement of strain and temperature using a fiber Bragg grating and a thermochromic material Sensors Actuators A 101 107-16

[17] Golnabi H 2002 Design of an optical fiber sensor for linear thermal expansion measurement Opt. Laser Technol. 34 389-94

[18] Fernández-Valdivielso C, Egozkue E, Matías I R, Arregui F J and Bariáin C 2003 Experimental study of a thermochromic material based optical fibre sensor for monitoring the temperature of the water in several applications Sensors Actuators B 91 231-40

[19] Black R J, Gonthier F, Lacroix S, Lapierre J and Bures J 1988 Tapered fibres: an overview Proc. SPIE 839 2-19

[20] Lacroix S, Bourbonnais R, Gonthier F and Bures J 1986 Tapered monomode optical fibers: understanding large power transfer Appl. Opt. 25 4421-5

[21] Díez A and Andrés M V 1996 Cylindrical multilayer optical waveguides: applications Proc. SPIE 2730 514-7

[22] Love J D et al 1991 Tapered single-mode fibres and devices, part 1: adiabaticity IEE Proc. J 138 343-54

[23] Birks T A and Li Y W 1992 The shape of fiber tapers J. Lightwave Technol. $10432-8$

[24] Kenny R K, Birks T A and Oakley K P 1991 Control of optical fiber taper shape Electron. Lett. 77 1654-6 